DOSSIÊ TEMÁTICO: Ensinar e aprender: metodologias e estratégias

d. https://doi.org/10.22481/praxisedu.v15i35.5670

\title{
DERECHOS Y OPORTUNIDADES EN LA ALFABETIZACIÓN INICIAL
}

\author{
RIGHTS AND OPPORTUNITIES IN INITIAL LITERACY
}

DIREITOS E OPORTUNIDADES NA ALFABETIZAÇÃO INICIAL

María Claudia Molinari

Universidad Nacional de La Plata - Argentina

\begin{abstract}
Resumen: El artículo analiza las decisiones político-pedagógicas en torno a la elección del objeto de enseñanza en momentos de la alfabetización inicial y a la valoración de los alumnos como ciudadanos portadores de saber, como personas con derecho a poner a prueba y transformar las conceptualizaciones no convencionales sobre la escritura en las situaciones de enseñanza. A fin de profundizar el sentido de estos enunciados, desde un marco constructivista se analizan textos producidos por alumnos de educación infantil y un fragmento de las interacciones en el aula en situación de escritura por los niños. La posibilidad de interpretar estos procesos de construcción conceptual en los alumnos es central para comprender el valor de las transformaciones en la escritura infantil y el tipo de problemas que los educadores necesitan plantear en las situaciones de enseñanza. Para finalizar, se explicitan algunas discusiones teóricas con la perspectiva de conciencia fonológica y con aportes de las neurociencias.
\end{abstract}

Palabras clave: Alfabetización inicial. Aprendizaje de la escritura. Enseñanza de la escritura.

\begin{abstract}
This article analyzes the political-pedagogical decisions concerning to the definition of the teaching object in initial literacy, and the recognition of students as knowledge-bearing citizens with the right to make reflections and transform unconventional concepts about writing in teaching situations. In order to deepen the meaning of these statements, from a constructivist perspective, we analyze the children's writings in early childhood education, and the registers of a classroom interactions when children were challenged to write. The interpret the student's conceptual construction processes is central to understand the value of children's writing transformation and the kind of problems that educators need to raise in teaching situations. Finally, we explicit some theoretical discussions on the phonological awareness perspective and the contributions of the neurosciences.
\end{abstract}

Keywords: Initial Literacy. Learning of Writing. Teaching Writing 
Resumo: $\mathrm{O}$ artigo analisa as decisões político-pedagógicas relativas a definição do objeto de ensino no momento da alfabetização inicial e o reconhecimento dos estudantes como cidadãos portadores de conhecimento, como pessoas com o direito de realizar reflexões e transformar conceitos não convencionais sobre a escrita em situações de ensino. A fim de aprofundar o sentido desses enunciados, a partir de uma perspectiva construtivista, analisa-se textos produzidos por crianças na educação infantil e um registro de interações ocorridas no contexto da sala de aula, em uma situação na qual as crianças são desafiadas a escrever. Interpretar esses processos de construção conceitual nos alunos é central para entender o valor das transformações na escrita infantil e o tipo de problemas que os educadores precisam levantar nas situações de ensino. Por fim, são explicitadas algumas discussões teóricas em torno da perspectiva da consciência fonológica e com contribuições das neurociências.

Palavras-chave: Alfabetização Inicial. Aprendizagem da Escrita. Ensino da Escrita.

\section{Introducción}

Toda diferencia puede ser considerada como déficit, depende de quién tenga el poder y de cómo lo use.

(LERNER, 2007, p. 7)

¿De qué hablamos cuando hablamos de alfabetización inicial? Nos referimos a un período en el que se enseñan y aprenden prácticas del lenguaje -prácticas sociales de lectura, escritura y oralidad- que circulan con sentidos diversos en las culturas de lo escrito y cuya apropiación es un derecho de todos los alumnos desde su ingreso al Jardín de Infantes y en los primeros años de la escolaridad primaria. ${ }^{1}$ Prácticas del leguaje en las que los alumnos exploran diversidad de géneros discursivos, al mismo tiempo que se apropian del funcionamiento del sistema de escritura convencional.

La enseñanza en los tiempos de la alfabetización inicial tiene un claro sentido político, en particular, en las decisiones acerca de cuál es el objeto de enseñanza así como en las decisiones acerca del lugar de los alumnos en las propuestas educativas.

Desde hace muchos años ha sido una decisión político-pedagógica que las prácticas del lenguaje sean el objeto de enseñanza fundamental desde los inicios de la alfabetización. Estas prácticas ponen en primer plano la manera en que utiliza el lenguaje para pensar, para producir ciertos efectos en los otros o incidir sobre las situaciones, para construir saberes; prácticas democratizadoras en tanto promueven la participación de todos los alumnos, donde la diversidad de usos y formas del lenguaje promueven en la escuela enriquecimiento

\footnotetext{
${ }^{1}$ Sin querer ocultar los usos sexistas del lenguaje, para facilitar la redacción y lectura en este documento se hace uso del genérico masculino para hacer referencia tanto a varones como a mujeres.
} 
lingüístico y no discriminación cultural. Prácticas que -desde muy pequeños- los niños pueden y deben ejercer para apropiarse del poder del lenguaje aunque inicialmente no logren hacerlo de forma convencional (PRE DISEÑO CURRICULAR PARA LA EDUCACIÓN GENERAL BÁSICA. Marco General, 1999).

También ha sido una decisión político-pedagógica considerar a nuestros alumnos como ciudadanos portadores de saber, como personas capaces y con "cronologías de aprendizaje diferentes" (TERIGI, 2010), con derecho a expresar y poner a prueba esos saberes en las situaciones de enseñanza, dudar de ellos y transformarlos con ayuda de intervenciones sistemáticas de sus maestros.

A fin de profundizar el sentido de estos enunciados se presentarán una selección de documentos recogidos en situaciones didácticas de escritura por los alumnos. El propósito es analizarlos desde un marco constructivista, a la vez que explicitar algunas de las discusiones vigentes en torno a la alfabetización inicial.

I-

Para planificar la enseñanza de las prácticas de lectura y escritura en Educación Inicial y en Educación Primaria seleccionamos algunos de los ámbitos en donde circula el lenguaje escrito en la práctica social, ámbitos vinculados a "diversas esferas de la actividad humana" (BAJTÍN, 1999, p. 248). Por ejemplo, situaciones didácticas de lectura y escritura en torno a lo literario, propuestas en ámbitos cotidianos y de estudio.

En el ámbito literario se planifican propuestas didácticas donde docentes y alumnos leen diversidad de géneros e intercambian sentidos en interacción con otros lectores. Al mismo tiempo, situaciones didácticas que invitan a escribir como lectores, por ejemplo, para comentar o recomendar obras de interés o para recrear personajes de los cuentos para una exposición.

Tal es el caso de las producciones sobre los lobos de los cuentos. En pequeños grupos los alumnos ilustran y componen textos descriptivos sobre el personaje. ${ }^{2}$

\footnotetext{
2 Grupo de 5 años- Docentes: Patricia Garelli y Laura Pereyra. Nivel Inicial, Escuela Graduada "Joaquín V. González". Universidad Nacional de La Plata (UNLP). La condición inicial para estas escrituras es haber participado de diversas situaciones de lectura en las que el lobo aparece como personaje.
} 


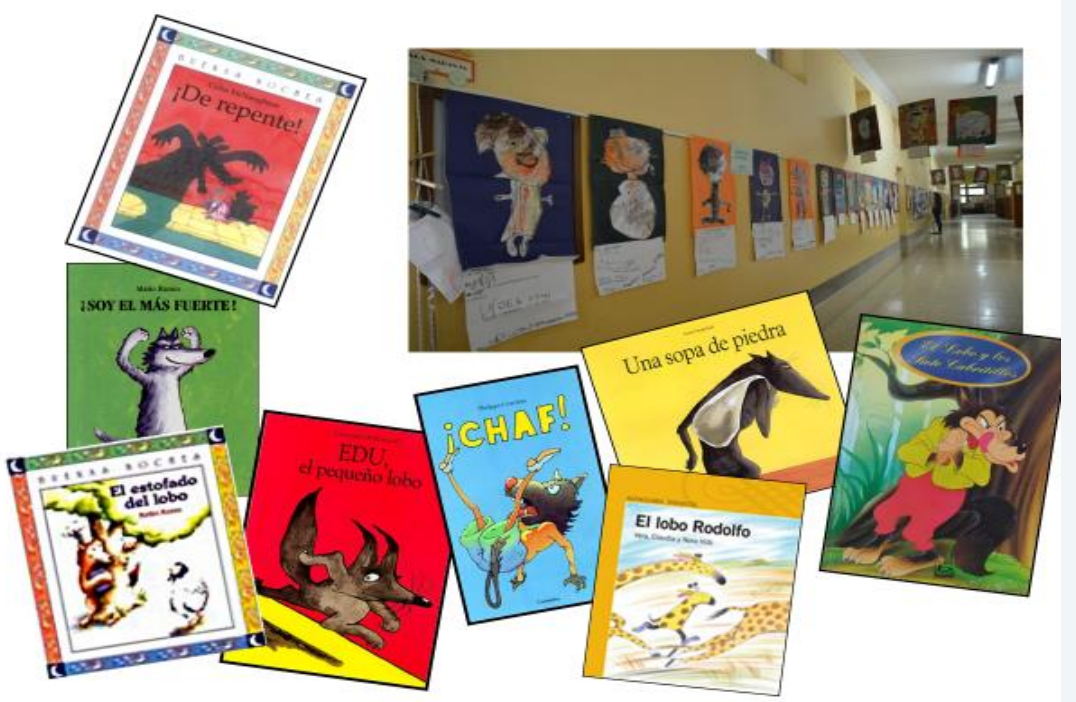

La escritura del lobo "Pedro" ("Se llama Pedro/es un lobo grande/ y peludo sus dientes son filosos y blancos") se resuelve de forma silábica, en la que se identifican algunos valores sonoros pertinentes.

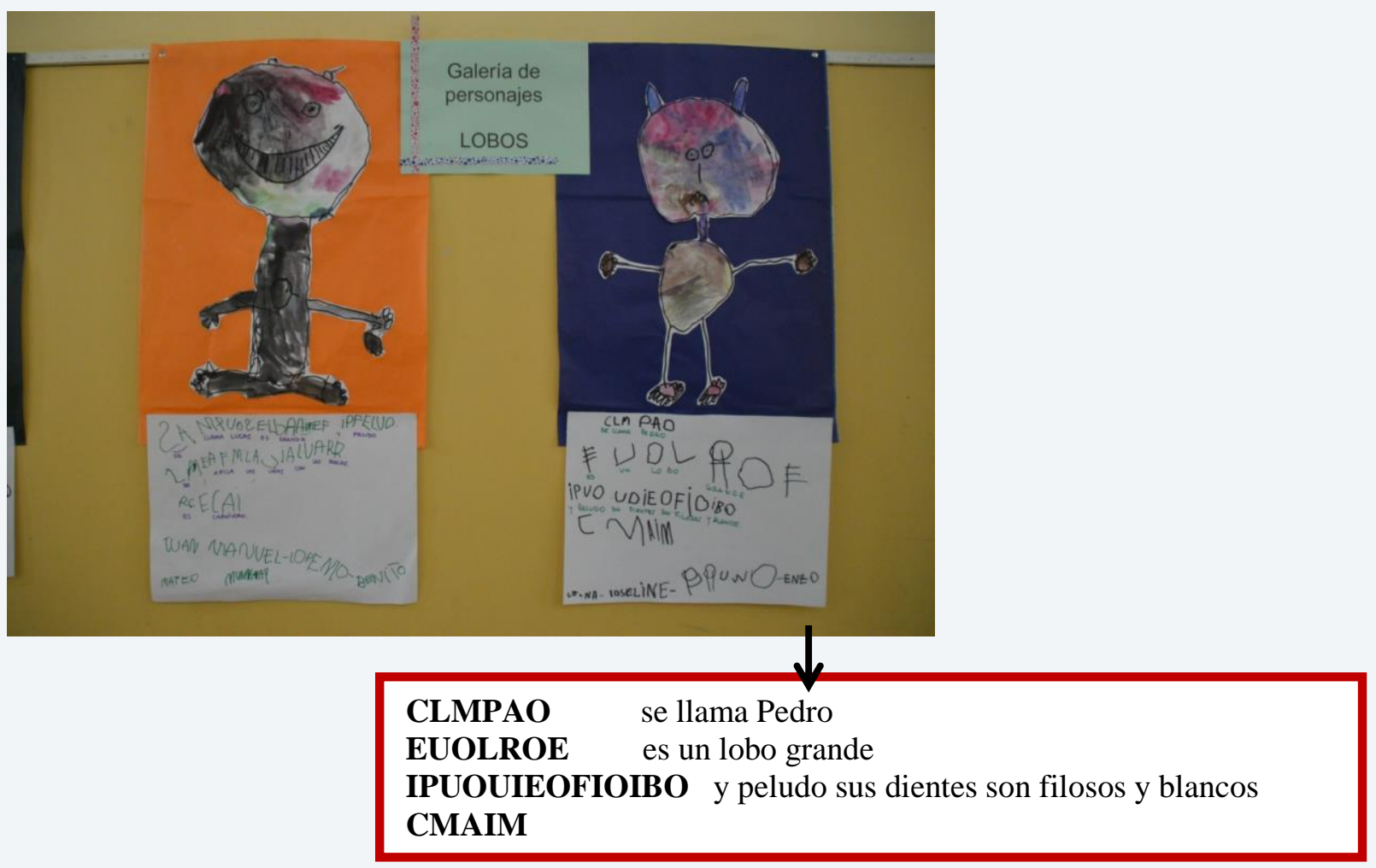

El texto del lobo "Umita” ("Se llama Umita/tiene fuerza y es feliz/usa collar/su cola es peluda y usa lentes") presenta cuatro líneas gráficas y -a diferencia del texto anterior- está escrito de manera presilábica. 


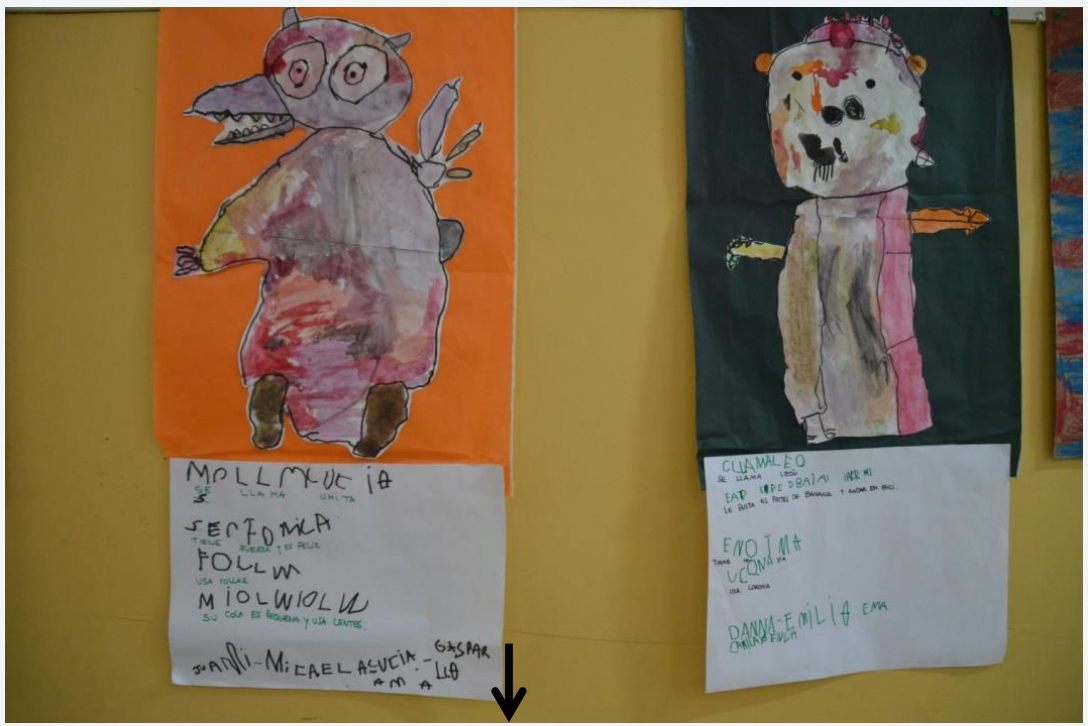

$\begin{array}{ll}\text { MOLLMLUCIA } & \text { Se llama Umita } \\ \text { SECFOMLA } & \text { tiene fuerza y es feliz } \\ \text { FOLLM } & \text { usa collar } \\ \text { MIOLWIOLN } & \text { su cola es peluda y usa lentes }\end{array}$

Como se advierte, aún desde producciones pre-fonetizantes, los alumnos pueden participar de procesos redaccionales complejos, compartiendo decisiones tanto sobre el lenguaje escrito como sobre el sistema de escritura en distintos niveles de conceptualización ${ }^{3}$.

II-

Las situaciones didácticas de lectura y escritura en ámbitos cotidianos nuclean prácticas del lenguaje vinculadas a la organización y al funcionamiento del aula y de la institución, así como la comunicación dentro y fuera de la escuela. Permiten anticipar o prever acciones, revisar y reflexionar sobre lo realizado, establecer diversas formas de intercambio escrito entre niños, docentes y otros adultos. Por ejemplo, registro de préstamo de libros de la biblioteca, diversas situaciones de escritura del nombre propio y de los compañeros en de agendas de cumpleaños o de teléfonos, producción de mensajes para destinatarios diversos, elaboración de juegos de mesa para la ludoteca de la institución (juegos de memoria, dominó, juego de cartas) (MOLINARI, 2015). Son prácticas del lenguaje similares a las que, a

\footnotetext{
${ }^{3}$ Desde hace tiempo el Jardín de Infantes ha asumido un compromiso alfabetizador. Sin embargo, resulta indispensable aclarar una vez más que "este compromiso no debe confundirse con la producción alfabética. No significa estipular niveles de logro por salas o edades, ni tener que adquirir la alfabeticidad del sistema de escritura al egreso del jardín “(MOLINARI, 2000, p. 19-20).
} 
propósito de la escritura, Bernad Lahire ha caracterizado desde la investigación sociológica como "prácticas de gestión doméstica":

[...] las prácticas de escritura de gestión doméstica permiten calcular, planificar, programar, prever la actividad y organizarla en un lapso más o menos largo, así como preparar o demorar la acción directa y suspender en parte la urgencia práctica. (LAHIRE, 2008, p. 9).

El cuaderno-agenda es un soporte donde los alumnos de forma individual o en pequeños grupos producen e interpretan escrituras con sentido social: listas de materiales, registro de una información que es necesario recordar, agendas de lectura literaria en las que registran o localizan títulos de cuentos leídos por la docente, etc. Es un cuaderno que acompaña a los niños desde que ingresan al Jardín de Infantes y continúa con ellos en primer año de la escuela primaria. Sus páginas conservan huellas de la historia personal de la escritura y sus conquistas en el proceso de construcción conceptual.

La producción de listas se presenta en todos los casos con sentido para los alumnos, en general, con el propósito de solicitar a la familia materiales necesarios para el desarrollo de alguna actividad. Las agendas de Camila y Manuela documentan sus conquistas en el tiempo.

\section{ESCRITURAS DE CAMILA}
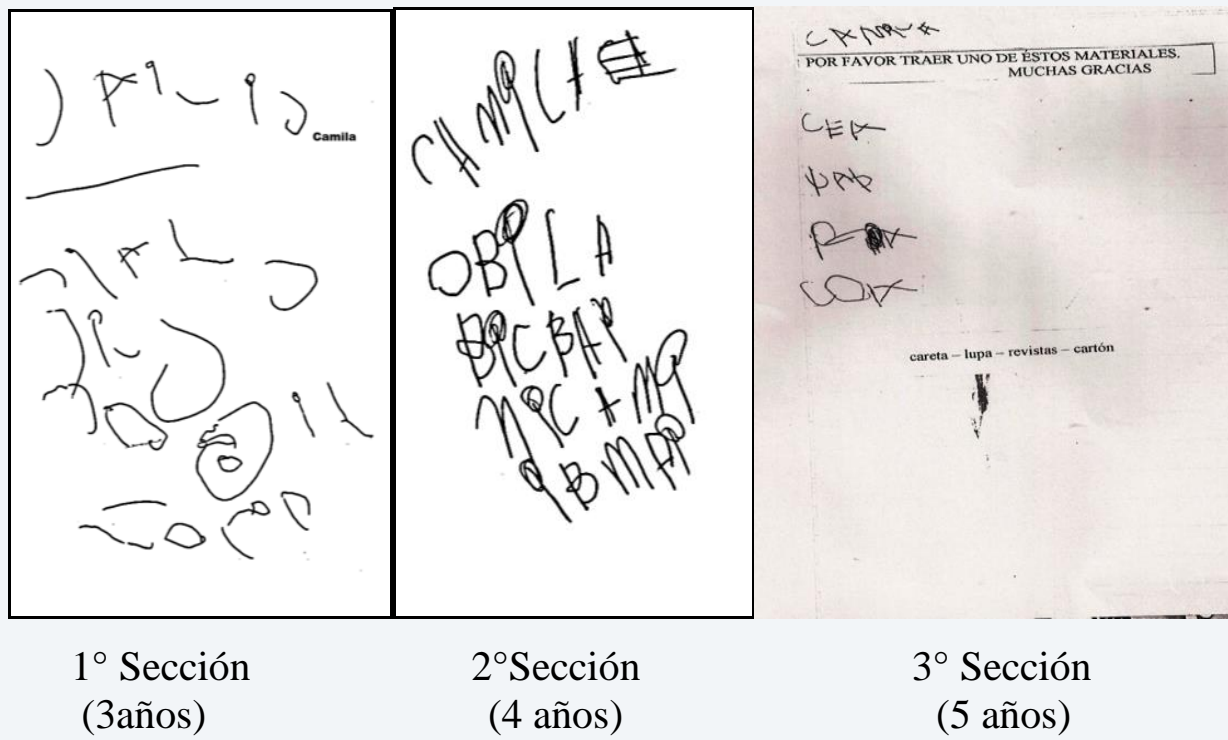

En las tres

listas escribe

CARETA

LUPA

REVISTAS

CARTÓN

Las listas escritas por Camila al finalizar las salas de 3, 4 y 5 años informan sobre el proceso de apropiación del sistema de escritura a lo largo del ciclo de Educación Inicial: escrituras presilábicas con grafismos primitivos (al finalizar la sala de 3 años), escrituras 
presilábicas diferenciadas (4 años) y escrituras silábicas con valores grafo-fónicos pertinentes (5 años). También la escritura del nombre propio presenta una interesante evolución.

En otro grupo de niños, las tres listas producidas por Manuela fueron escritas en el transcurso del último año escolar (sala de 5 años) ${ }^{4}$.

\section{ESCRITURAS DE MANUELA}

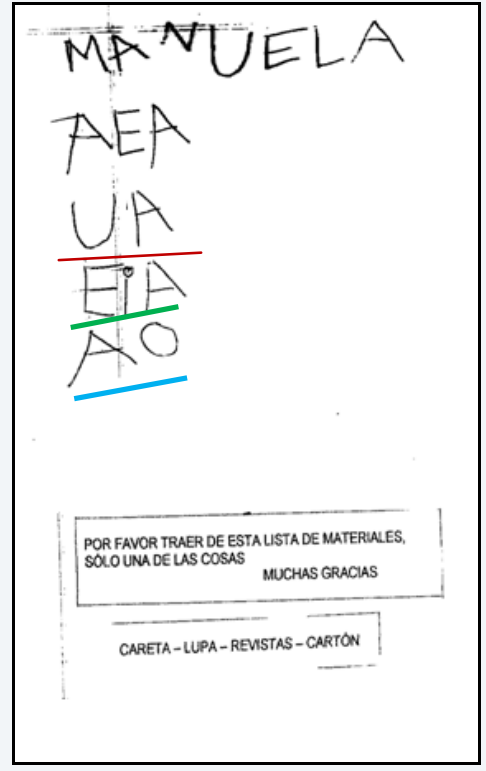

Marzo

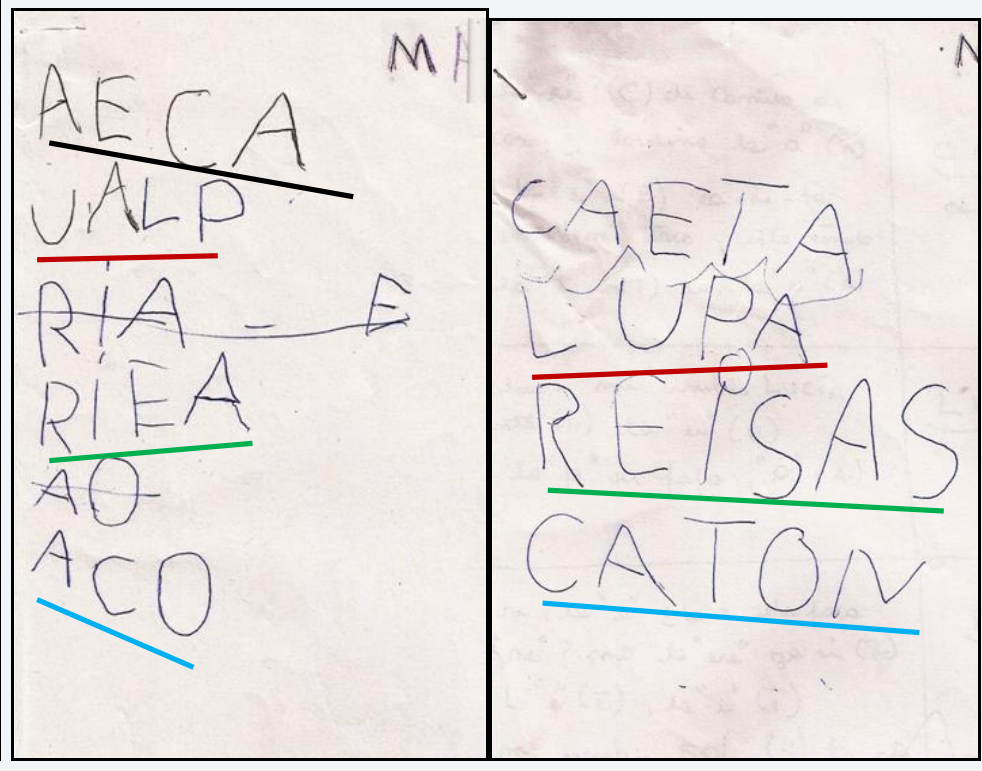

Agosto

Noviembre

\section{CARETA \\ LUPA \\ REVISTAS \\ CARTÓN \\ AEA/AECA/CAETA \\ UA/UALP/LUPA \\ EIA/ RIEA/ REISAS \\ $\mathrm{AO} / \mathrm{ACO} / \mathrm{CATON}$}

En las producciones inicial, media y final se registran avances importantes en la construcción del sistema a de escritura. En el mes de marzo Manuela escribe de forma silábica con valores grafo-fónicos pertinentes en vocales y en noviembre produce la lista de materiales con escrituras casi alfabéticas.

Analicemos en particular "revistas" (EIA/ RIEA/ REISAS) y "cartón" (AO/ ACO / CATON). La producción intermedia correspondiente al mes de agosto. Es un claro ejemplo de los problemas y soluciones provisorias de los niños cuando advierten la necesidad de introducir más letras pertinentes en su escritura. Como claramente ocurre en "revistas"

\footnotetext{
${ }^{4}$ Las docentes pueden proponer la escritura de listas diferentes, o bien, conservar las mismas palabras como en las escrituras de Camila y Manuela. En ambos casos es posible analizar la evolución de la escritura y desde estos datos tomar decisiones de enseñanza en el transcurso del año escolar (Jardín de Infantes Nuestra Sra. Del Valle, La Plata. Coordinación: Prof. Andrea Ocampo)
} 
(RIEA) y en "cartón" (ACO), el "desorden con pertinencia" en la escritura es un fenómeno esperable en el proceso de aprendizaje (FERREIRO, 2009).

En ambas palabras Manuela produce una primera versión que, al ser interpretadas, decide realizar un nuevo intento: de la escritura inicial RIA ( una letra pertinente por sílaba para "re-vis-tas") reescribe RIEA al incorporar E para que diga "re" ; en "cartón", de la primera versión AO ( una vocal por sílaba para "car-tón"), reescribe ACO pues para "car" advierte que necesita agregar una letra más (AC ). Algo similar sucede en "careta" cuando incorpora C para "ca" (AECA).

Según aportes de Ferreiro, este desorden con pertinencia testimonia la manera en que por análisis de la sílaba- los niños incorporan más letras pertinentes en su escrito, aunque sin poder resolver de manera inmediata su ubicación correcta en la serie gráfica (FERREIRO, 2009). Las producciones de Manuela (revistas-cartón-careta) deben ser interpretadas como intentos claros de avance conceptual, en tanto explora cómo introducir más de una unidad grafo-fónica pertinente por sílaba, hecho que hasta no hace mucho resultaba inadmisible.

En "lupa" (UALP) Manuela produce una escritura diferente: reescribe de forma silábica dos veces la misma palabra ante la necesidad de agregar- por hipótesis de cantidad mínima- más de dos letras en "lu-pa" (UA). Produce dos escrituras silábicas contiguas que no logra interpretar: primero con vocales (lu-pa: UA) y luego con consonantes (lu -pa: LP). Escribe solo una letra por sílaba pero desde dos anclajes diferentes: cuando dice "' $1 u^{\prime}$ 'lu' 'lu' 'la u' ", escribe U; cuando repite “' $1 u^{\prime}$ ' $1 u^{\prime} / 1 / / 1 /$ " , escribe L. (FERREIRO, 2009). ${ }^{5}$ Es interesante constatar que la niña conoce las cuatro letras de "lupa" -las identifica y las puede graficar- pero esta información no alcanza para comprender el funcionamiento alfabético de la escritura. En este caso, es la hipótesis silábica la que guía la producción.

Los maestros que comprenden la complejidad de estos procesos saben que la inclusión de unidades intra-silábicas no sucede de forma lineal por adición o simple agregado de letras pertinentes en orden convencional (FERREIRO, 2009). Por el contrario, saben que en el proceso de reorganización del sistema anterior -en este caso el silábico- el desorden de las letras es una respuesta esperable y avanzada en el proceso de alfabetización. Los alumnos descubren que ciertas unidades grafo-fónicas deben ser incorporadas en su escritura, pero no comprenden de forma inmediata en qué lugar de la serie las deben introducir. Los docentes que interpretan el sentido de estas respuestas impulsan en el aula su discusión.

\footnotetext{
${ }^{5}$ La escritura de una letra por sílaba desde dos anclajes diferentes, también ha sido analizada en la producción de alternancias grafo-fónicas en el nivel silábico de escritura (MOLINARI; FERREIRO, 2007; TERUGGI; MOLINARI, 2015).
} 
La posibilidad de interpretar los procesos de construcción conceptual en los niños es central para comprender el valor de las transformaciones en la escritura infantil y el tipo de problemas que los educadores necesitan plantear en las situaciones de enseñanza. Consideremos la palabra "cartón" (ACO) escrita por Manuela a fin de ejemplificar posibles intervenciones de los docentes:

- aportar información a través de la escritura de otras palabras ("Como vos dijiste, 'carta' y 'carpa' empiezan como 'cartón'. Las escribo (CARTA-CARPA) y vamos a pensar cómo dice 'cartón' en tu escritura" (ACO).

- Recurrir a escrituras conocidas como los nombres propios, a fin de localizar información para producir otras escrituras ("Traé el cartel donde dice "Carla", allí hay información que te puede servir para escribir 'cartón'”).

- Favorecer el intercambio entre pares para pensar juntos durante el proceso de producción (“Cada uno de ustedes escribió “cartón”, pero como vemos, las escrituras tienen algunas diferencias. Piensen juntos cómo dice en cada caso, decidiendo qué dejarían o cambiarían y escriban entre los dos nuevamente "cartón). (MOLINARI; CORRAL, 2008).

Interpretar y valorar las escrituras pre-alfabéticas, supone considerar los procesos de apropiación como conquista de los alumnos en el contexto escolar y no como versiones deficitarias con respecto a la escritura convencional. Al comprender aquellos procesos los educadores plantean situaciones didácticas desafiantes e intervenciones profesionales complejas donde los alumnos tienen oportunidades de tomar decisiones acerca de cuáles letras escribir, cuántas y en qué orden.

III-

En este apartado, se analizan algunos pasajes de las interacciones en el aula, en este caso, en el marco de situaciones didáctica de lectura y escritura en ámbitos de estudio o "para saber más”. En ellas se ofrece a los alumnos oportunidades para enfrentar la complejidad de textos informativos a través de la lectura de la maestra y por lectura de los niños, tomar algunas notas para conservar datos de interés y escribir por dictando a la docente y/o por los alumnos textos para publicar y difundir información.

La producción de infografías sobre animales polares es un ejemplo de este tipo propuesta. $^{6}$ En parejas, los alumnos escriben epígrafes en un conjunto de imágenes

\footnotetext{
${ }^{6}$ Proyecto publicado en: MOLINARI; BRENA; OCAMPO, 2015.
} 
recuperando algunos conocimientos sobre las características de los animales y las condiciones del ambiente donde habitan. La situación de escritura de epígrafes comienza con la anticipación del texto que cada pareja se propone escribir ${ }^{7}$. En otra clase, las parejas escriben aquellos textos de forma colaborativa. Estas decisiones didácticas en la secuencia de producción, ofrece mejores oportunidades para que los alumnos puedan resolver problemas diferentes en tiempos diferentes: primero la composición del escrito (sobre qué escribir y cómo enunciarlo en lengua escrita para que otro comprenda) y luego su textualización, donde el foco de los intercambios sucede en torno al sistema de escritura.

Previo a la producción, la docente recuerda que hay escrituras disponibles que pueden consultar "cuando necesiten saber más sobre las letras" (ejemplo, los nombres todos los compañeros), que se trata de una tarea colaborativa con intercambio de roles y que recibirán su ayuda cuando recorra los distintos grupos.

Para analizar el funcionamiento de la situación de producción se presenta un fragmento de clase en el que Clarita y Milagros escriben sobre el krill. En este breve pasaje interesa destacar las interacciones en el proceso de escritura, en particular, la manera en que las autoras toman decisiones sobre las letras.

Las niñas habían previsto escribir "son patas plumosas para cazar algas" a propósito de una imagen donde se destacan las patas delanteras del krill (patas con plumosidades que utiliza como filtro del alimento y cuya función es la de cazar algas).

Mientras la docente se desplaza y trabaja con otros grupos, Clarita y Milagros han escrito:

\section{PAMESPACAS}

\section{LAUSOPA}

C
$\mathrm{Al}$ acercarse a las niñas, la docente observa la escritura y solicita interpretación.
$[\ldots]$

El libro analiza de forma pormenorizada la puesta en aula de una propuesta de desarrollo curricular diseñada, coordinada y documentada por Andrea Ocampo y Graciela Brena. Docente: Noelia Zavala, Jardín de Infantes $\mathrm{N}^{\circ}$ 907, Provincia de Buenos Aires, Argentina.

${ }^{7}$ Se hace referencia aquí al momento de la composición oral del texto. Hablar para escribir -según Ana Camps (1992) - implica una situación diferente de la que se establece entre interlocutores en una conversación, pues se trata de enunciados formulados oralmente para ser escritos. En otros términos, propuestas orales de texto escrito que la autora ha definido como "texto intentado" y que -durante la interacción- el grupo puede someterlas a consideración antes de darle forma gráfica. Durante este proceso, el texto intentado puede experimentar cambios, reformulaciones o revisiones a lo largo del proceso de composición (al igual que sucede en el texto escrito). (CAMPS; GUASCH; MILLAN; RIVAS, 2007). 
D: Lean ¿Todo junto, cómo era?

(Milagros lee mientras señala en el texto)

Milagros: 'Son patas plumosas' (señala PAMESPACAS) 'para’ (señala LAUSOPA)

'ca...' (señala C para 'cazar' )

Clarita: /s/,s/, /“caz' /s/ la 'ese'.

(Milagros agrega S y queda CS. Vuelve a leer hasta la tercera línea gráfica. En CS se detiene a pensar).

D: 'Son patas plumosas para...'

Milagros y Clarita: 'Cazar algas' (completando la frase).

$[\ldots]$

Clarita: 'Algas', 'al', 'gas'/s/ la 'a' (escribe a continuación y queda CSA).
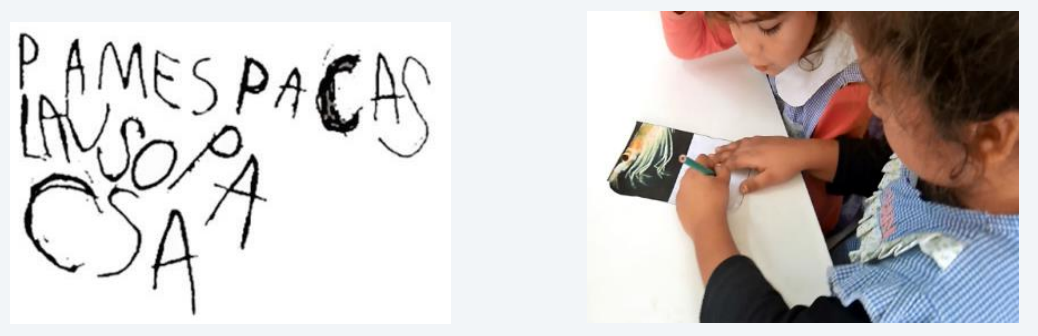

Clarita: La 'ese', la 'ce'

Milagros: ¡Esperá!

Clarita: 'Para'... (señalando ambas el texto).

Milagros: 'Ca', /s/ (en CSA).

Clarita: ¿La 'ese'?

(Milagros asiente con la cabeza).

Clarita: ¡Otra vez la ‘ese’ ahí! ... ‘ese’ (escribe S y queda CSAS).

D: Léanlo.

(Ambas leen mientras Milagros señala con el dedo).

D: Ustedes dijeron que decía 'son patas plumosas para cazar algas'. ¿Dónde dice 'patas'?

Milagros y Clarita: ¡Acá! (señalan PACAS).

D: ¿Dónde empieza y dónde termina 'patas'?

(Clarita subraya PACAS).

D: ¿Dónde dice ‘son'?

Milagros y Clarita: ¡Acá! (señalan PAMES).

D: Digan 'son'. 
Clarita: 'Son', la 'ese' (escribe S sobre P). 'Son, son' (escribe N sobre A), 'son' la 'o' (escribe $\mathrm{O}$ arriba de $\mathrm{M}$, queda entonces reescrito $\mathrm{SNO}$ ).

Milagros: No, primero ésta, acá (señala $\mathrm{O}$ en SNO, indicando que va antes que N).

Clarita: No, después la 'o’.

Milagros: 'Son' (en SNO) 'patas' (PACAS). (Relee por propia iniciativa).

D: ¿Y éstas que no señalaron? (indica ES en SNOESPACAS).

(Milagros tacha ES y vuelve a releer en cada línea gráfica).

$[\ldots]$

\section{Primera escritura}

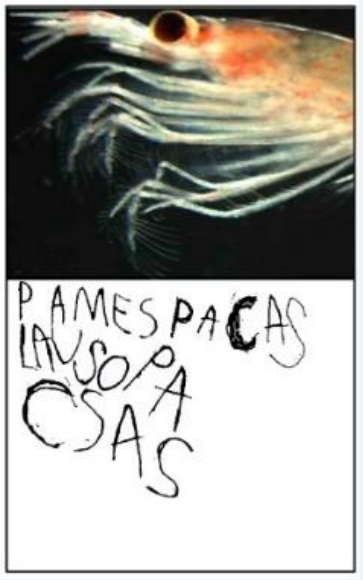

Escritura final

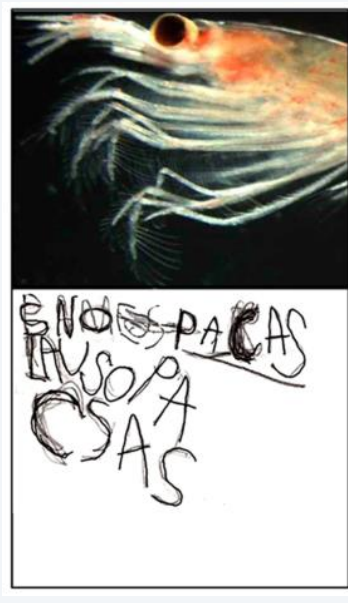

"son patas /plumosas para /cazar algas"

A partir de intervenciones de la docente, el control de la propia escritura aparece aquí como una práctica de escritor que las niñas ejercen en su ausencia, de manera cada vez más autónoma. Para las autoras parece necesario estar seguras de cuáles y cuántas letras escribir para que diga aquello que se han propuesto comunicar.

Por momentos, la maestra se acerca y les solicita que interpreten su producción a fin de comprender cómo las niñas han resuelto su la escritura, y a partir de esta constatación, plantearles algunos problemas. Con este propósito, les solicita localizar algunos segmentos (“¿Dónde dice ‘patas”?”, “Dónde empieza y dónde termina ‘patas’?”, “Dónde dice ‘son””); les propone reescribir "son" invitando a que exploren relaciones entre oralidad y escritura ("digan son"); les solicita también ajustar la escritura tachando un sobrante que no han logrado interpretar ( ES en SNOESPACAS para que diga "son -SON- patas -PACAS" ). La docente regula sus intervenciones, atendiendo al interés y compromiso de las alumnas en la situación de producción. Son intervenciones de enseñanza acotadas que focalizan solo en algún o en algunos problemas en los cuales los pequeños tienen oportunidades de pensar y aprender. 
Considerando los conocimientos sobre el sistema de escritura, la producción presenta resoluciones silábicas a la vez que intentos más o menos ajustados de introducir unidades fonológicas más pequeñas en la sílaba. Las niñas han avanzado hacia una producción silábicoalfabética, en la que el desorden (SNO para "son") se conserva a pesar de las indicaciones de Milagros para desplazar una de sus letras.

Clarita y Milagros producen de forma colaborativa. Llama la atención la manera en que logran intercambiar información durante el proceso, alternando el rol de escritor. Al iniciar la tarea saben qué texto van a escribir ('son patas plumosas para cazar algas') y en el transcurso de la escritura recuperan dicho texto una y otra vez. Durante este proceso, exploran relaciones entre segmentaciones de la emisión oral para identificar y escribir unidades grafo-fónicas pertinentes, en un proceso dialéctico de decir-escribir/escribir-decir; al mismo tiempo, exploran relaciones entre emisión oral y partes de la escritura para interpretar y controlar lo que ya han escrito. Por ejemplo, en "cazar" luego de escribir C interpretada como "ca", Clarita verbaliza "/s/, /s/, "caz'/s/ la "ese"”, indicando a Milagros que debe escribir S para "zar" (queda escrito C ='ca', S ='zar'). De forma similar sucede en "algas" y también en "son", en un proceso que puede caracterizarse como de "oralidad analítica" y de “escritura reflexiva” (FERREIRO, 2009, p. 12).

Resulta importante señalar que en este proceso la maestra no promueve el deletreo o el silabeo desde su intervención; solo invita a las niñas a decir lo que van a reescribir -“son"en el momento de reformular esta escritura. Es necesario destacar que las segmentaciones de la oralidad son procedimientos propuestos y explorados por las niñas para pensar la escritura en el transcurso de la producción.

En este proceso es posible observar relaciones entre el nivel de conceptualización de la escritura y las capacidades de segmentación oral en momentos de decidir qué unidades escribir y de justificar lo escrito. Desde hace mucho tiempo, resultados de investigación han constatado esta relación, en discusión con la perspectiva de conciencia fonológica (VERNON, 1997; VERNON; FERREIRO, 1999). Las autoras informan:

[...] que los niveles de conceptualización de la escritura [...] correlacionan con las capacidades de segmentación de la oralidad disponibles en ese momento del desarrollo. Adicionalmente, sugieren que la segmentación de la emisión oral puede surgir como respuesta a un problema cognitivo (la justificación, para sí mismo o para un interlocutor, de las partes de una palabra escrita) y no necesita ser planteado como un entrenamiento de una habilidad fuera de contexto. (FERREIRO, 2002, p. 159 /el destacado es nuestro). 
IV-

En las situaciones didácticas expuestas se ha analizado cómo -desde los inicios de la alfabetización- todos los alumnos pueden componer textos aproximándose al lenguaje escrito en diversidad de géneros, escribir palabras pero también textos más extensos en situaciones con sentido social, escribir y poner en discusión el sistema de ideas que construyen cuando intentan comprender el funcionamiento de la escritura.

Desde hace décadas, investigaciones y experiencias didácticas sistematizadas han aportado un valioso material teórico sobre la enseñanza y el aprendizaje escolar desde la perspectiva expuesta (LERNER; PALACIOS DE PIZANI; MUÑOZ DE PIMENTEL, 1987; KAUFMAN, 1988; KAUFMAN; CASTEDO; TERUGGI; MOLINARI, 1989; LERNER, 2001; NEMIROVSKY, 1999; TEBEROSKY, 1992; TOLCHINSKY, 1993; WEISZ, 1999). El análisis pormenorizado del funcionamiento de situaciones didácticas en jardines de infantes y escuelas, ha sido y sigue siendo fundamental para comprender la complejidad de las interacciones del aula en tiempos de la alfabetización inicial. Investigaciones comparativas entre propuestas de enseñanza y logros de aprendizaje en los niños al finalizar el Jardín de Infantes, reportan mejores resultados en quienes tuvieron oportunidades de escribir en situaciones similares a las expuestas en este trabajo. ${ }^{8}$

Sin duda, el tiempo de la alfabetización inicial es un campo polémico, en particular, cuando se discute sobre la enseñanza y el aprendizaje del sistema de escritura. Las propuestas de alfabetización fundadas en la perspectiva de conciencia fonológica (phonological awareness), en consonancia con los aportes de las neurociencias, ingresan sin dificultad a la dinámica homogeneizadora de la escuela con prescripciones de enseñanza garantes del éxito escolar (DEFIOR, 1996; DIUK, 2013; BORZONE; MARDER, 2015; DEHAENE, 2015; DEHAENE, 2014). Cuando los alumnos no logran aprender con estos programas de enseñanza caracterizados como de intervención "fuerte" - RAI, "Respuesta a la Intervención"“puede pensarse en un trastorno específico de aprendizaje" (BORZONE; MARDER, 2015, p. 25). Sin poner en discusión el dispositivo de enseñanza, queda claro que el déficit sigue siendo propiedad del alumno.

\footnotetext{
8. Los resultados de aprendizaje en grupos de niños de cinco años de sectores populares argentinos y brasileños al finalizar el Nivel Inicial, que participaron de prácticas de enseñanza diferentes -a quienes se les propuso entre otras tareas la escritura de listas de palabras- concluyen que, quienes participaron de prácticas de enseñanza constructivistas, alcanzaron niveles de escritura más avanzados y recurrían a procedimientos más elaborados que aquellos niños que participaron de propuestas basadas en actividades perceptivo-motrices y enseñanza graduada de letras. (GRUNFELD: 2012; SCARPA, 2014; GRUNFELD; MOLINARI, 2017; GRUNFELD; SCARPA, 2018).
} 
La difusión de los aportes de las neurociencias al campo educativo sobre el aprendizaje y la enseñanza de la lectura, informa desde una perspectiva reduccionista al campo de lo biológico y de lo individual (TERIGI, 2016) “cómo aprende el cerebro a leer" (DEHAENE, 2015, p. 21), las consecuencias de ese aprendizaje en el cerebro, los retrasos en la lectura en contextos de pobreza o la detección temprana de patologías en niños muy pequeños...

En materiales escolares para jardines de infantes y escuelas primarias, se diseñan actividades en las que -como condición inicial- promueven la enseñanza de habilidades perceptivo- motoras y habilidades orales de toma de conciencia fonológica para identificar y deslindar sonidos en palabras -a modo aprestamiento- para luego enseñar de forma explícita y graduada las correspondencias entre fonemas y grafemas a todo el grupo escolar (DIUK, 2013; BORZONE; MARDER, 2015; ROSEMBERG; STEIN, 2015). Así, la escritura y la lectura se enseñan como mecanismos de trascripción entre unidades sonoras y unidades gráficas (MORAIS, 2014; MALUF; CARDOSO-MARTINS, 2013), desde una interpretación monocrónica del aprendizaje ${ }^{9}$ (TERIGI, 2010) a alumnos desprovistos de todo saber.

La posibilidad de que los alumnos puedan expresar sus conocimientos como lectores y escritores en las situaciones de enseñanza es un derecho que por decisión política se impulsa desde una perspectiva constructivista. En la formación de ciudadanos críticos y autónomos, este derecho es una condición fundamental.

\section{REFERÊNCIAS}

BAJTÍN, M. Estética de la creación verbal. México, Siglo XXI. 1999.

BORZONE, A.M; MARDER, S. Leamos juntos. Guía del docente. Buenos Aires, Paidós. 2015.

CAMPS, A.; GUASCH, O.; MILLAN, M.; RIVAS, T. "El escrito en la oralidad: el texto intentado", en: Archivos de Ciencias de la Educación. Departamento de Ciencias de la Educación. FaHCE, UNLP, año 1, no 1, 2007.

DEFIOR, S, "Una clasificación de las tareas utilizadas en la evaluación de las habilidades fonológicas y algunas ideas para su mejora”. Revista Infancia y Aprendizaje, Journal for the Study of Education and Development, no 73, 1996.

\footnotetext{
${ }^{9}$ En palabras de Flavia Terigi, "cuando hablamos de aprendizajes monocrónicos estamos hablando de la idea de que es necesario proponer una secuencia única de aprendizajes para todos los miembros del grupo escolar y sostener esta secuencia en el tiempo de modo que al final de un proceso de enseñanza el grupo de alumnos hayan logrado aprender las mismas cosas" (TERIGI, 2010, p. 16).
} 
DEHAENE, S. El cerebro lector. Últimas noticias de las neurociencias sobre lectura, la enseñanza, el aprendizaje y la dislexia. México: Siglo Veintiuno Editores. 2014.

DEHAENE, S. Aprender a leer. De las ciencias cognitivas al aula. Bs As: Siglo veintiuno editores. 2015.

DIUK, B. Propuesta Dale! Derecho a aprender a leer y escribir. Guía para el docente. Fundación Perez Companc y ETIS. 2013.

FERREIRO, E. "La desestabilización de las escrituras silábicas: alternancia y desorden con pertinencia". En Lectura y Vida, Revista Latinoamericana de Lectura. Año 30, 2, 6-13. 2009.

FERREIRO, E. Escritura y oralidad: unidades, niveles de análisis y conciencia metalingüística. En FERREIRO, E. (comp.). Relaciones de (in)dependencia entre oralidad y escritura, 151-171. Barcelona: Gedisa. 2002.

GRUNFELD, D. "La palabra escrita y la palabra oral al final de la sala de 5 años. Contraste entre dos propuestas de enseñanza en escuelas públicas”. Tesis de Maestría. Facultad de Humanidades y Ciencias de la Educación, UNLP. 2012. Disponible en: http://sedici.unlp.edu.ar/handle/10915/31815.

GRUNFELD, D.; MOLINARI, M. C. La importancia de pensar sobre la escritura en el jardín de infantes. Magis, Revista Internacional de Investigación en Educación, 9 (19), 31-48. 2017.

GRUNFELD, D.; SCARPA, R. Comprender las unidades menores de la palabra. Un desafío crucial en la alfabetización inicial. Investigaciones en la Argentina y Brasil. Archivos de Ciencias de la Educación, vol. 12, núm. 13, 2018 Universidad Nacional de La Plata, Argentina. 2018.

KAUFMAN, A. M. La lectoescritura y la escuela. Una experiencia constructivista. Buenos Aires: Santillana. 1988.

KAUFMAN, A. M.; CASTEDO, M.; TERUGGI, L.; MOLINARI, M. C. Alfabetización de niños: construcción e intercambio. Buenos Aires: Aique. 1989.

LAHIRE, B. Escrituras domésticas. La domesticación de lo doméstico. Lectura y Vida, Año 29, 3. 2008.

LERNER, D. “Enseñar en la diversidad”. Lectura y Vida. Año 28,4 (p.6-17). 2007.

LERNER, D. Leer y escribir en la escuela: lo real, lo posible y lo necesario. Ciudad de México: Fondo de Cultura Económica, FCE. 2001

LERNER, D.; PALACIOS DE PIZANI, A.; MUÑOZ DE PIMENTEL, M. Comprensión de la lectura y expresión escrita en niños alfabetizados. Serie de publicaciones DEE-OEA (cuatro fascículos). Caracas: Ministerio de Educación, Dirección de Educación Especial, DEE. 1987. 
MALUF, M.R; CARDOSO-MARTINS, C. (org). Alfabetização no século XXI. Como se aprende a ler e a escrever. Porto Alegre, Penso Editora. 2013.

MOLINARI, M. C. "Escritura y revisión en la producción de juegos de mesa" en Quehacer Educativo, No 131 (Junio), pp. 64-71. Montevideo: FUM-TEP (reedición), 2015.

MOLINARI, M. C.; BRENA, G.; OCAMPO, A. Ambiente Natural y Social y Prácticas del Lenguaje. Análisis de un proyecto sobre animales. Orientaciones didácticas para el Nivel Inicial. $7^{\circ}$-Materiales complementarios: documentos fotográficos y fragmentos de video de clase. 2015. Disponible en:

http://servicios2.abc.gov.ar/lainstitucion/sistemaeducativo/educacioninicial/proyectoanimales/ index.html

MOLINARI, M. C.; CORRAL, A. La escritura en la alfabetización inicial. Producir con otros en la escuela y en el jardín. La Plata: DGCyE de la Provincia de Buenos Aires. 2008. Disponible en:

http://servicios2.abc.gov.ar/lainstitucion/organismos/lecturayescritura/default.cfm

MOLINARI, M. C. “Leer y escribir en el jardín de infantes”. En A. M. Kaufman (comp) Letras y números. Alternativas didácticas para el Jardín de Infantes y Primer Ciclo de EGB. Buenos Aires: Santillana. 2000.

MOLINARI, M. C.; FERREIRO, Emilia. Identidades y diferencias en las primeras etapas del proceso de alfabetización. Escrituras realizadas en papel y en computadora. Lectura y Vida, Revista Latinoamericana de Lectura, 28 (4), 18-30. 2007.

MORAIS, J. Alfabetizar para a democracia. Porto Alegre, Penso Editora. 2014.

NEMIROVSKY, M. Sobre la enseñanza del lenguaje escrito... y temas aledaños. Ciudad de México: Paidós. 1999.

PRE DISEÑO CURRICULAR PARA LA EDUCACIÓN GENERAL BÁSICA. Marco General. Dirección de Currículo. Secretaría de Educación, Gobierno de la Ciudad de Buenos Aires, 1999.

ROSEMBERG, C. R.; STEIN, A. La alfabetización temprana en el nivel inicial. Juegos con sonidos, rimas, letras y poesías para promover el aprendizaje del sistema de escritura. Guía 3. Ciudad Autónoma de Buenos Aires: OEI- UNICEF. 2015.

SCARPA, R. L. P. O conhecimento de pré-escolares sobre a escrita: impactos de propostas didáticas diferentes em regiões vulneráveis. (Tese Doutorado - Programa de Pós-Graduação em Educação. Área de Concentração: Didática, Teorias de Ensino e Práticas Escolares). Universidade de São Paulo, Faculdade de Educação, São Paulo. 2014. Disponible en: http://www.teses.usp.br/teses/disponiveis/48/48134/tde-20102014-115756/pt-br.php

TEBEROSKY, A. Aprendiendo a escribir. Barcelona: Instituto de Ciencias de la Educación de la Universidad de Barcelona, ICE, Horsori Editorial. 1992. 
TERIGI, F. "Las cronologías de aprendizaje: un concepto para pensar las trayectorias escolares". Conferencia dirigida a directores y supervisores escolares de la Provincia de La Pampa en la Jornada de Apertura Ciclo Lectivo 2010. Santa Rosa (La Pampa), 22 y 23 de febrero de 2010. Disponible en:

http://www.lapampa.edu.ar:4040/bicentenario/index.php/acciones-educativas/ciclo-de-cine-yconferencias/cronologiasdeaprendizaje.html

TERIGI, F. "Sobre aprendizaje escolar y neurociencias". Propuesta Educativa. Número 46, Año 25,Vol 2 p. 50- 64. 2016.

TERUGGI, L.; MOLINARI, C. Analisi comparativa del fenomeno dell'alternanza grafofonica nella scrittura manoscritta e digitale di bambini di lingua spagnola e italiana di 4 e 5 anni. Rassegna di Psicologia, 32 (2), 11-29. 2015. Disponible en:

http://www.memoria.fahce.unlp.edu.ar/art_revistas/pr.7255/pr.7255.pdf

TOLCHINSKY, L. Aprendizaje del lenguaje escrito: procesos evolutivos e implicaciones didácticas. Barcelona: Universidad Pedagógica Nacional de México, Secretaría de Educación Pública de México, SEP, Editorial Anthropos. 1993.

VERNON, S.; FERREIRO, Emilia. "Writing development. A neglecded variable in the consideration of phonological awareness". Harvard Educational Review, vol. 69, núm.4, p.359-415. 1999.

VERNON, S. La relación entre la conciencia fonológica y los niveles de conceptualización de la escritura. Tesis de doctorado. México: Departamento de Investigaciones Educativas, Centro de Investigación y de Estudios Avanzados del Instituto Pedagógico Nacional, DIE-CINVESTAV, 1997.

WEISZ, T. O diálogo entre o ensino e a aprendizagem. São Paulo: Editora Atica, 1999.

\section{SOBRE A AUTORA:}

\section{María Claudia Molinari}

Profesora en Ciencias de la Educación, Facultad de Humanidades y Ciencias de la Educación, Universidad Nacional de La Plata, República Argentina. DIE-CINVESTAV- México. E-mail: cmolinari@ fahce.unlp.edu.ar

(iD) http://orcid.org/0000-0002-5129-8500 